\title{
Chimeric tumor modeling reveals role of partial PDL1 expression in resistance to virally induced immunotherapy
}

\author{
Mee Y. Bartee, Parker C. Dryja and Eric Bartee* (D)
}

\begin{abstract}
Expression of PDL1 on the surface of tumor cells can blunt the efficacy of many cancer immunotherapies. For example, our lab has previously shown that tumors derived from malignant cells incapable of expressing PDL1 are highly susceptible to immunotherapy induced by oncolytic virus treatment while tumors derived from PDL1 capable cells are highly resistant. In patient biopsies, however, expression of PDL1 on malignant cells is often not uniform with some cells expressing PDL1 while others do not. Importantly, how this partial PDL1 positivity influences the outcomes of immunotherapy remains largely unknown. In the current work, we expand on our previous findings by generating partially PDL1 positive tumors in immune competent animals and asking what percentage of tumor cells must express PDL1 for a tumor to become functionally resistant to oncolytic treatment. Our results indicate that the responsiveness of partially PDL $1^{+}$tumors correlates linearly with the percentage of PDL1 capable cells present at the initiation of treatment. Additionally, we observe that tumors which relapse after treatment display a significant increase in the numbers of PDL1 capable cells present suggesting that specific editing of mixed tumors might play a role in disease relapse. These data indicate that varying levels of PDL1 expression can play a significant role in the outcomes of oncolytic immunotherapy and challenges the concept that tumors should be viewed as simply PDL1 ${ }^{+}$or PDL1 ${ }^{-}$.
\end{abstract}

Keywords: Immunotherapy, Oncolytic virotherapy, Myxoma virus, Tumor heterogeneity, PDL1

\section{Introduction}

Programmed cell death protein 1 (PD1) is an immune checkpoint protein which is rapidly expressed on $\mathrm{T}$ cells following their activation. Engagement of PD1 with its primary ligand, programmed death ligand 1 (PDL1), results in $\mathrm{T}$ cell dysfunction and eventual exhaustion [1,2]. This pathway exists to maintain peripheral tolerance and prevent autoimmunity, however, overexpression of PDL1 within the tumor microenvironment is seen in a variety of malignancies and likely represents a major mechanism through which cancer escapes immune surveillance [3, 4]. While PDL1 can be found on a variety of cell types within the tumor microenvironment [5-10], expression is most frequently observed on malignant tumor cells themselves were it can range from completely $\mathrm{PDL1}^{-}$to virtually $100 \%$

\footnotetext{
* Correspondence: bartee@musc.edu

Department of Microbiology and Immunology, Medical University of South Carolina, Basic Science Building Rm 208C, 173 Ashley Ave, Charleston, SC 29425, USA
}

(c) The Author(s). 2019 Open Access This article is distributed under the terms of the Creative Commons Attribution 4.0 International License (http://creativecommons.org/licenses/by/4.0/), which permits unrestricted use, distribution, and reproduction in any medium, provided you give appropriate credit to the original author(s) and the source, provide a link to the Creative Commons license, and indicate if changes were made. The Creative Commons Public Domain Dedication waiver (http://creativecommons.org/publicdomain/zero/1.0/) applies to the data made available in this article, unless otherwise stated.
PDL1 $^{+}[11-15]$. In patients whose tumor cells are PDL1 positive, this expression displays two distinct patterns [16]. The first, inducible expression, presents as a spatially separated PDL1 $1^{-}$core surrounded by $\mathrm{PDL} 1^{+}$cells and results from upregulation of PDL1 by IFNY expressed from tumor infiltrating lymphocytes (TIL) $[17,18]$. The second pattern, constitutive expression, presents as tumors which varying mixtures of both PDL1 ${ }^{+}$and PDL1 ${ }^{-}$cells and is caused by mutations, such as EGFR activation [19] or loss of PTEN function [20], within individual cells. Despite this complex intratumoral expression pattern, there is not a consensus on how many PDL1 expressing tumor cells are required to constitute a functionally PDL ${ }^{+}$tumor. Therefore, identifying how expression of PDL1 on specific percentages of tumor cells impacts the outcomes of immunotherapy, and what constitutes a functionally $\mathrm{PDL}^{+}{ }^{+}$tumor, remain critical unanswered questions.

Oncolytic virotherapy (OV) is a form of localized immunotherapy in which injection of a cancer-tropic virus 
results in the generation of potent anti-tumor $\mathrm{T}$ cell responses $[21,22]$. We have previously demonstrated that tumors which are incapable of expressing PDL1 on their malignant cells are highly susceptible to OV while those which express high levels of PDL1 are highly resistant [23]. In the current manuscript, we now expand on our previous findings by using a novel method of chimeric tumor modeling to generate partially $\mathrm{PDL}^{+}$tumors in immune competent animals and study how this partial PDL1 positivity impacts the outcomes of virally induced immunotherapy.

\section{Results}

\section{Generation of PDL1-chimeric tumors}

Our previous studies have demonstrated that the presence of PDL1 on tumor cells plays a significant role in determining the outcomes of MYXV-based OV ( [23] and Additional file 1: Figures S1 and S2). However, while PDL1 is expressed on a high percentage of malignant cells in most preclinical models [23], analysis of primary tumor biopsies has revealed a much more complex pattern of expression $[12,24]$. In order to assess how this partial PDL1 positivity impacted oncolytic immunotherapy, we therefore used chimeric tumor modeling to generate tumors in vivo which contained various percentages of $\mathrm{PDL}^{+}$cells. B16/F10 ${ }^{\text {scramble }}$ and B16/ F10 ${ }^{\text {PDL1-l- }}$ cells were mixed ex vivo at either a 1:1 or 1:9 ratio and the mixtures then injected into C57/B6 mice (Fig. 1a). As controls, pure cultures of either B16/ $\mathrm{F} 10^{\text {scramble }}$ or B16/F10 $10^{\mathrm{PDL} 1-1-}$ cells were injected in parallel. To assess the success of this strategy at generating partially PDL1 positive tumors, animals were euthanized 8 days after injection and the capacity of tumor cells to express PDL1 was analyzed (Fig. 1b). Consistent with the formation of PDL1-uniform tumors, animals injected with pure cultures of B16/F10 $0^{\text {scramble }}$ cells developed tumors which were capable of expressing PDL1 on the vast majority of malignant cells (78.2\% PDL1 capable) while animals injected with pure cultures of B16/ F10 ${ }^{\text {PDL1-/- }}$ cells developed tumors which were virtually devoid of PDL1 expressing malignant cells (2.1\% PDL1 capable). In contrast, animals injected with a 1:1 ratio of $\mathrm{B} 16 / \mathrm{F} 10^{\text {scramble }}$ to $\mathrm{B} 16 / \mathrm{F} 10^{\mathrm{PDL} 1-/-}$ cells developed tumors which were capable of expressing PDL1 on $46 \%$ of their tumor cells, while animals injected with a 1:9 ratio developed tumors capable of expressing PDL1 on $4.9 \%$ of their tumor cells(Fig. 1b). These data suggest that the tumors resulting from injection of mixtures of PDL1 capable and PDL1 deficient cells correlate linearly with the cellular input. Injection of different mixtures of PDL1 capable and PDL1 deficient cells did not impact the expression of PDL1 on tumor infiltrating lymphocytes (TIL) which were uniformly capable of expressing this protein regardless of the makeup of the injected malignant cells (Fig. 1c).
Partial PDL1 positivity linearly impacts the outcomes of oncolytic immunotherapy

We next asked how our PDL1 chimeric tumors would respond to OV with the hypothesis that they would either separate into discrete $\mathrm{PDL}^{+}$and $\mathrm{PDL}^{-}$groups or display a more linear dose response. Tumors generated from injection of either a $1: 1$ or $1: 9$ mixture of $\mathrm{B} 16 / \mathrm{F} 10^{\text {scramble }}$ and B16/F10 ${ }^{\text {PDL1-I- }}$ cells were established for 7 days and then treated with MYXV. Tumors established from injection of pure $\mathrm{B} 16 / \mathrm{F} 10^{\text {scramble }}$ or $\mathrm{B} 16 / \mathrm{F} 10^{\mathrm{PDL} 1-/-}$ cells were also included as controls. Consistent with previous results treatment of fully PDL1 deficient tumors resulted in significant tumor regression $(80 \%, 12 / 15$ mice) while treatment of fully PDL1 capable tumors resulted in only short term stable disease. Treatment of both 1:1 and 1:9 chimeric tumors resulted in intermediate phenotypes with some animals displaying tumor regression and others displayed stable disease followed by relapse. When the outcomes of all animals were taken into account, the overall therapeutic efficacy of MYXV treatment correlated strongly with the percentage of PDL1 capable cells found in a tumor at the time of treatment with 1:1 chimeric tumors responding better then fully PDL1 capable tumors but not as well as 1:9 chimeric tumors (Figs $2 \mathrm{a}-\mathrm{c}$ ). These data suggest that tumors expressing PDL1 in a limited number of tumor cells will likely relapse following OV, however, these tumors will still gain more benefit from therapy than tumors expressing PDL1 on a high percentage of malignant cells.

\section{Partially PDL1 positive tumors display specific immunoediting of $\mathrm{PDL}^{-/-}$cells}

To begin to understand why chimeric tumors displayed such reduced responsiveness to MYXV treatment, we next attempted to identify major differences between homogenous and mixed tumors before and after relapse. Phenotypically, chimeric tumors resulting from injection of a 1:9 ratio of B16/F10 $0^{\text {scramble }}$ to B16/F10 ${ }^{\text {PDL1-/- }}$ cells appeared to be similar to tumors generated from injection of pure $\mathrm{B} 16 / \mathrm{F} 10^{\mathrm{PDL1}-/-}$ cells, displaying a slightly reduced growth rate compared to pure B16/F10 $0^{\text {scramble }}$ tumors (Fig. 3a-c). Interestingly however, 8 days after implantation these chimeric tumors were immunologically distinct from both $\mathrm{B} 16 / \mathrm{F} 10^{\text {scramble }}$ and B16/F10 ${ }^{\mathrm{PDL} 1-/-}$ tumors displaying the increased infiltration of total lymphocytes $\left(\mathrm{CD} 45^{+}\right.$cells) seen in PDL1 deficient tumors but the reduced numbers of $\mathrm{CD}^{+}$ and $\mathrm{T}_{\text {con }}$ cells typically seen in PDL1 capable tumors (Fig. 3d). Interestingly, both $\mathrm{CD}^{+}$and $\mathrm{T}_{\text {con }}$ cells found in chimeric tumors had also significantly increased expression of CD69 compared to both PDL1 deficient and capable tumors, suggesting that partial PDL1 positivity might influence both the immunological makeup and activity of tumors.

Somewhat surprisingly, however, we found that while PDL1 capable, PDL1 deficient, and 1:9 chimeric tumors were immunologically distinct prior to oncolytic treatment, 


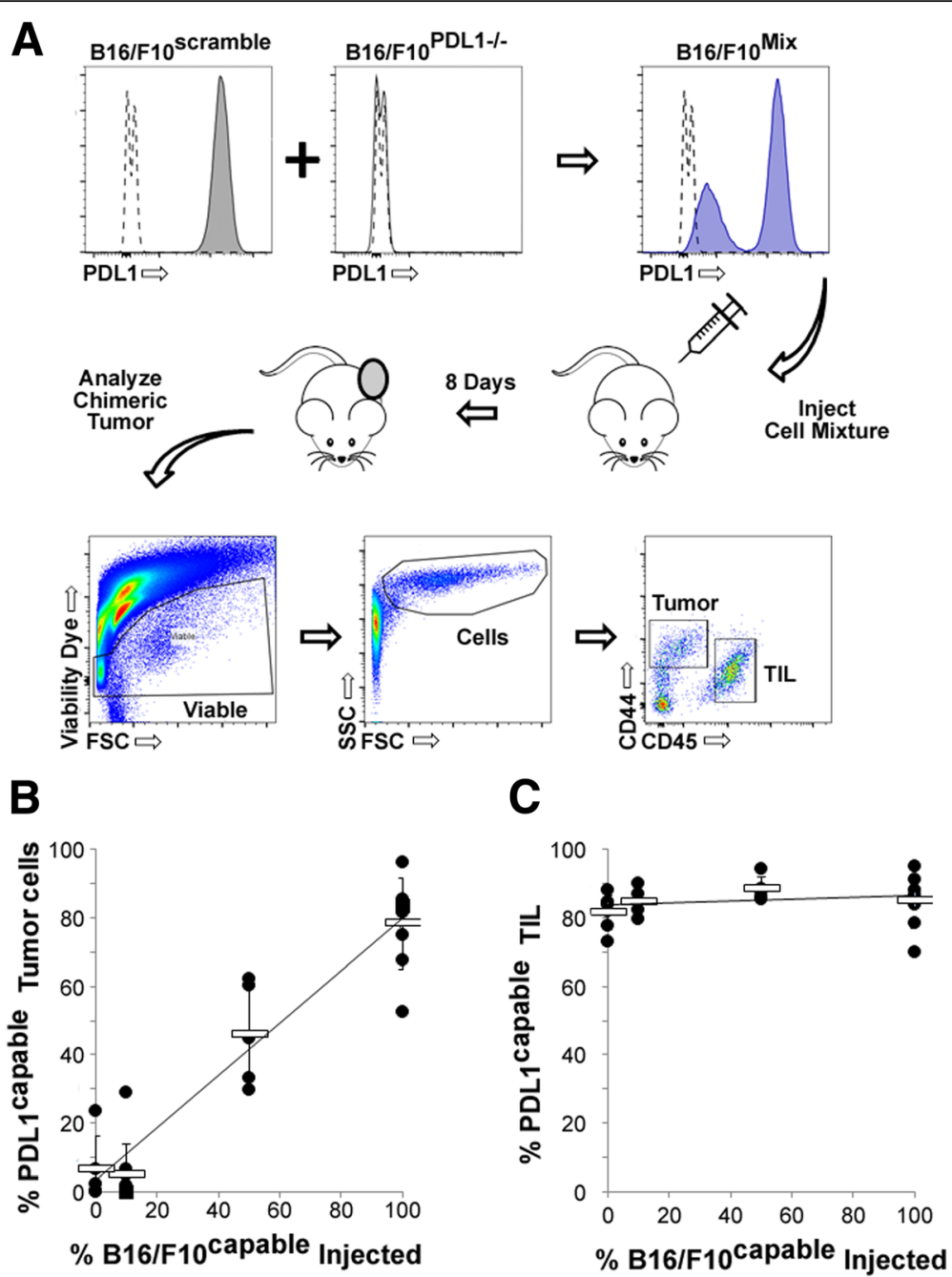

Fig. 1 Generation of PDL1 Chimeric Tumors. a Schematic representation of generation and analysis of in vivo chimeric tumors. b Average percent of PDL1 capable tumor cells found in tumors initially generated from the indicated cellular makeup. Data represents summation of two independent experiments. c Average percent of PDL1 capable TIL found in tumors initially generated from the indicated cellular makeup. Data represents summation of two independent experiments

all of these tumor types displayed similar immunological signatures following OV (our unpublished observations). In contrast, analysis of the malignant cells found in each tumor before and after treatment revealed that relapsing chimeric tumors displayed a striking change in makeup. Consistent with our previous findings, immediately prior to the initiation of therapy 1:9 chimeric tumors were comprised of $\sim 5-10 \%$ PDL1 capable cells. Following relapse, however, the percent of PDL1 capable cells found in these tumors had increased to comprise the vast majority of malignant cells recovered (Fig. 4a). This change in phenotype appeared specific to chimeric tumors, since tumors derived from pure cultures of either B16/F10 $0^{\text {scramble }}$ or B16/ F10 ${ }^{\text {PDL1-/- }}$ cells retained their initial makeup following therapy. No obvious differences in the growth rates of $\mathrm{B} 16 / \mathrm{F} 10^{\text {scramble }}$ or B16/F10 ${ }^{\text {PDL1-/- }}$ cells could be seen in vitro (Fig 4b and c) and both PDL1 ${ }^{+}$and $\mathrm{PDL}^{-/-}$cells were equally sensitive to MYXV infection (Additional file 1: Figure S3) suggesting that editing was not caused by differences in either inherent cell growth or sensitivity to viral infection. In contrast, editing occurred to a significantly lesser extent in immune deficient NOD/Scid mice (Fig. 4d) and could also be inhibited by depletion of either: $\mathrm{CD}^{+}$and NK1.1 $1^{+}$cells (Fig. 4e) or the specific CD8 ${ }^{+}$cytotoxic lymphocyte population (Fig. 4f). We therefore hypothesize that the change in phenotype seen in our mixed tumors following relapse represents a specific immunoediting of PDL1 ${ }^{-/}$tumor cells.

\section{Discussion}

An increasing number of studies have demonstrated that the PD1/PDL1 checkpoint pathway plays a significant 


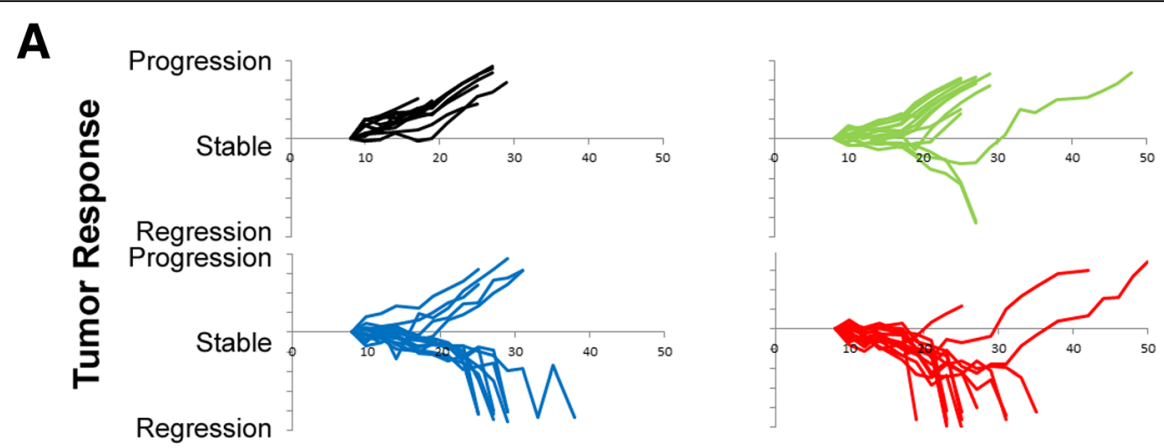

Days Post Treatment

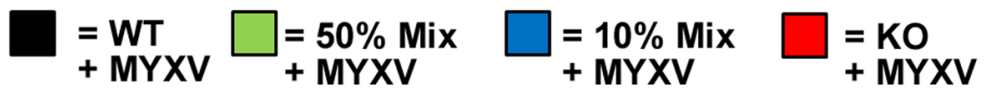

B

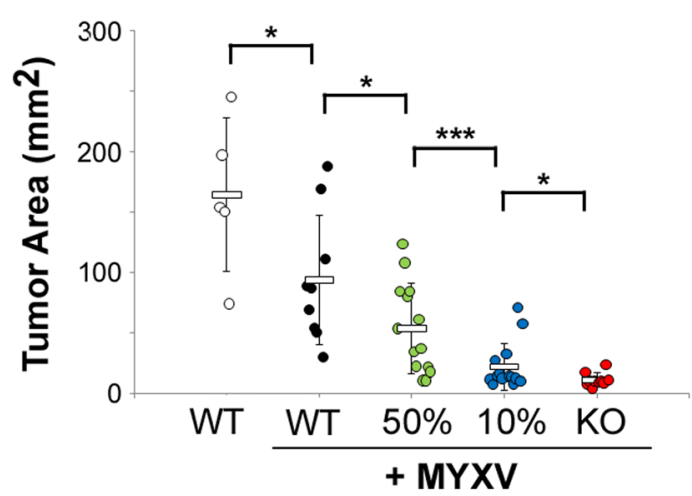

C

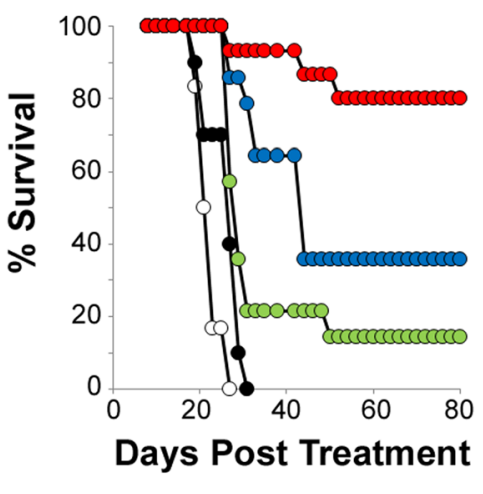

Fig. 2 PDL1 Chimeric tumors are resistant to oncolytic therapy. a-d $4 \times 10^{5}$ total cells comprising pure cultures of either B16/F10 $0^{\text {scramble }}(\mathrm{WT}: n=$ $10)$, or B16/F10 PDL1-/- $(\mathrm{KO}: n=15)$, or either a 1:9 mix $(10 \% n=14)$, or a 1:1 mix $(50 \% \mathrm{n}=14)$ of WT to KO cells were injected SQ into the left flank of syngeneic C57/B6 mice. 7, 9, and 11 days post tumor implantation; tumors were treated with IT injection of $1 \times 10^{7}$ foci forming units of MYXV. Tumors were then monitored every other day for tumor growth and animals euthanized when tumors reached $15 \mathrm{~mm}$ in any direction. a Response of individual tumors to treatment. Data is displayed as percent tumor area ( $\mathrm{LxW}$ ) compared to tumor area immediately prior to initiation of treatment. b Tumor area (LxW) in individual mice at day 20 post tumor cell injection. Statistical significance was determined using unpaired students T-Test $\left(*<0.05,{ }^{* *}<0.01\right)$. c Overall survival animal survival. Data represents summation of two individual experiments

role in determining the efficacy of OV $[21-23,25]$. These studies, however, have used systemically injected PD1 blocking reagents to identify potential therapeutic synergies with oncolytic viruses and are therefore unable to address the specific impact of PDL1 expressed on various cell types. In contrast, in our current work we specifically eliminated PDL1 expression on malignant cells and demonstrate that tumors derived from these cells are highly susceptible to OV (Additional file 1: Figures S1 and S2). Interestingly, these tumors are not completely devoid of PDL1 as they contain relatively high numbers of $\mathrm{CD} 45^{+} / \mathrm{PDL}^{+}$TIL (Fig. 3). These data therefore specifically implicate the PDL1 expressed on malignant cells as playing a highly significant role in the efficacy of OV. There are, however, several potential caveats to this conclusion. First, while our data clearly shows that tumors which fail to express PDL1 on malignant cells are susceptible to OV, it does not specifically demonstrate that the PDL1 expressed on TIL is not negatively impacting therapy. Indeed, it is possible that elimination of PDL1 from TIL would further enhance the efficacy of virotherapy. Alternatively, recent studies have shown that PDL1 expressed on either tumor cells or TIL can play significantly different roles in different models $[26,27]$. Thus, it is possible that the high sensitivity of PDL1 deficient tumors to OV has to do with our use of the B16/F10 model. Further experiments are therefore needed to better clarify the role of tumor and TIL expressed PDL1 during OV.

The primary purpose of this work was to study the impact of partial PDL1 positivity on OV. To accomplish this, we utilized CRISPR/Cas9 technology to generate a matched set of PDL1 capable and PDL1 deficient cell lines which were then mixed ex vivo and injected into animals to form PDL1 chimeric tumors (Fig. 1). This methodology represents a relatively novel use of CRISPR 


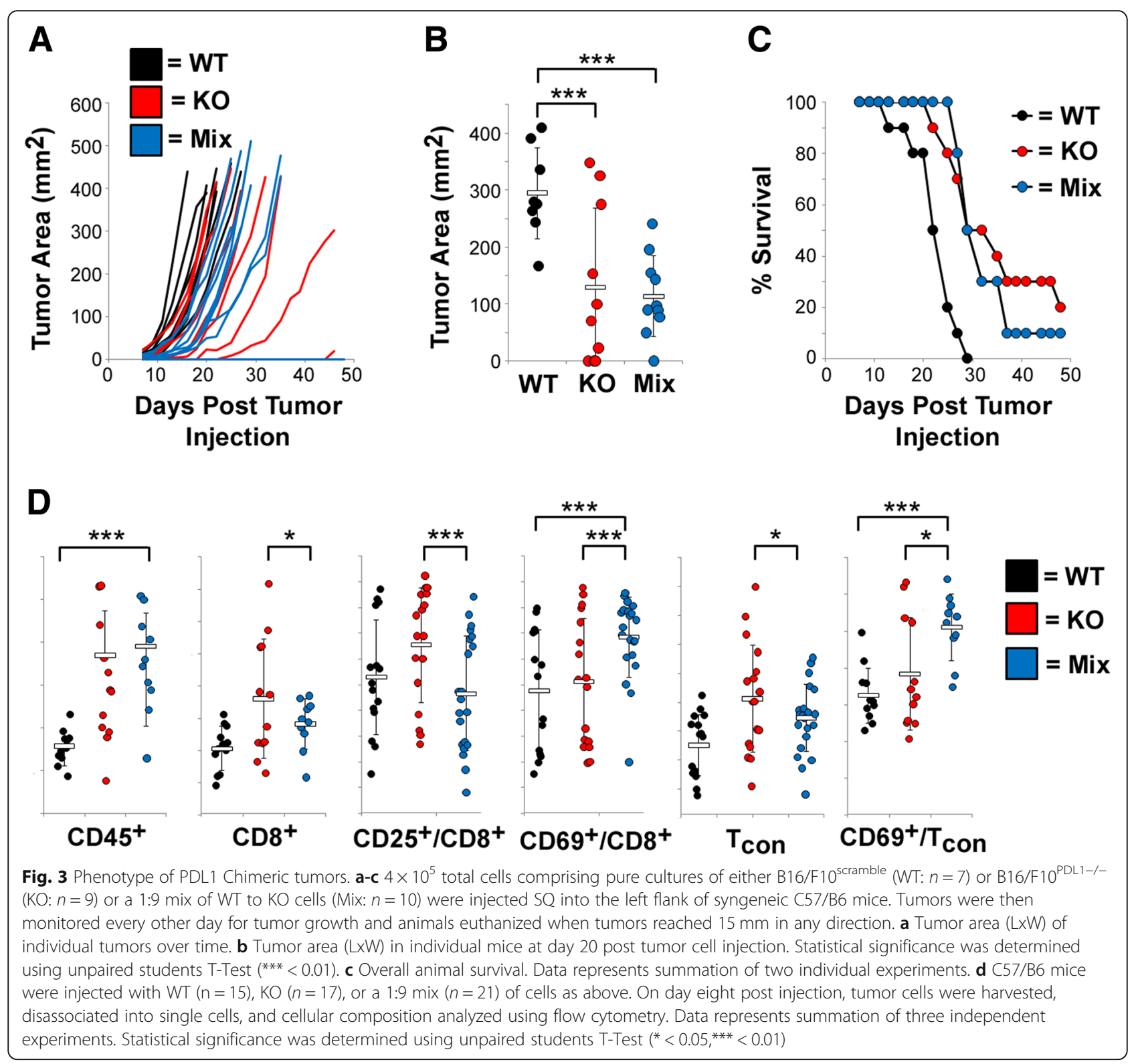

technology which we feel has a number of significant uses in cancer research, including: loss of tumor antigens, mixed susceptibility to viral infection, mixed expression of tumor mutations, and dominance of various tumor phenotypes. Our studies, however, reveal several key factors which much be taken into consideration when conducting these types of studies. First is that the parent and daughter cell lines used must establish tumors with a linear correlation to the input mixture of cells. In our experiments, loss of PDL1 did not significantly impact the establishment of tumors in C57/B6 mice (Fig. 3). However, this is unlikely to be true for all proteins. If loss of a given protein significantly impacts tumor establishment, then the chimeric tumors resulting from injection of cell mixtures might have highly variable makeups which will complicate interpretation of results. Second is that it is highly beneficial if the cell lines used can be easily distinguished following tumor excision. For surface antigens, such as PDL1, this can be accomplished using flow cytometry, although care must be taken in identification of the tumor cells. Alternatively, tagging each cell line with a unique fluorophore (ie GFP vs RFP) might suffice, although care should be taken to account for differential immunogenicity if this approach is used.

Our results with this novel mixed tumor model demonstrate that even expression of PDL1 on a limited number of tumors cells can significantly impact the outcomes of therapy, causing relapse of $50-60 \%$ of tumors in the B16/F10 model (Fig. 2). Additionally, we found 


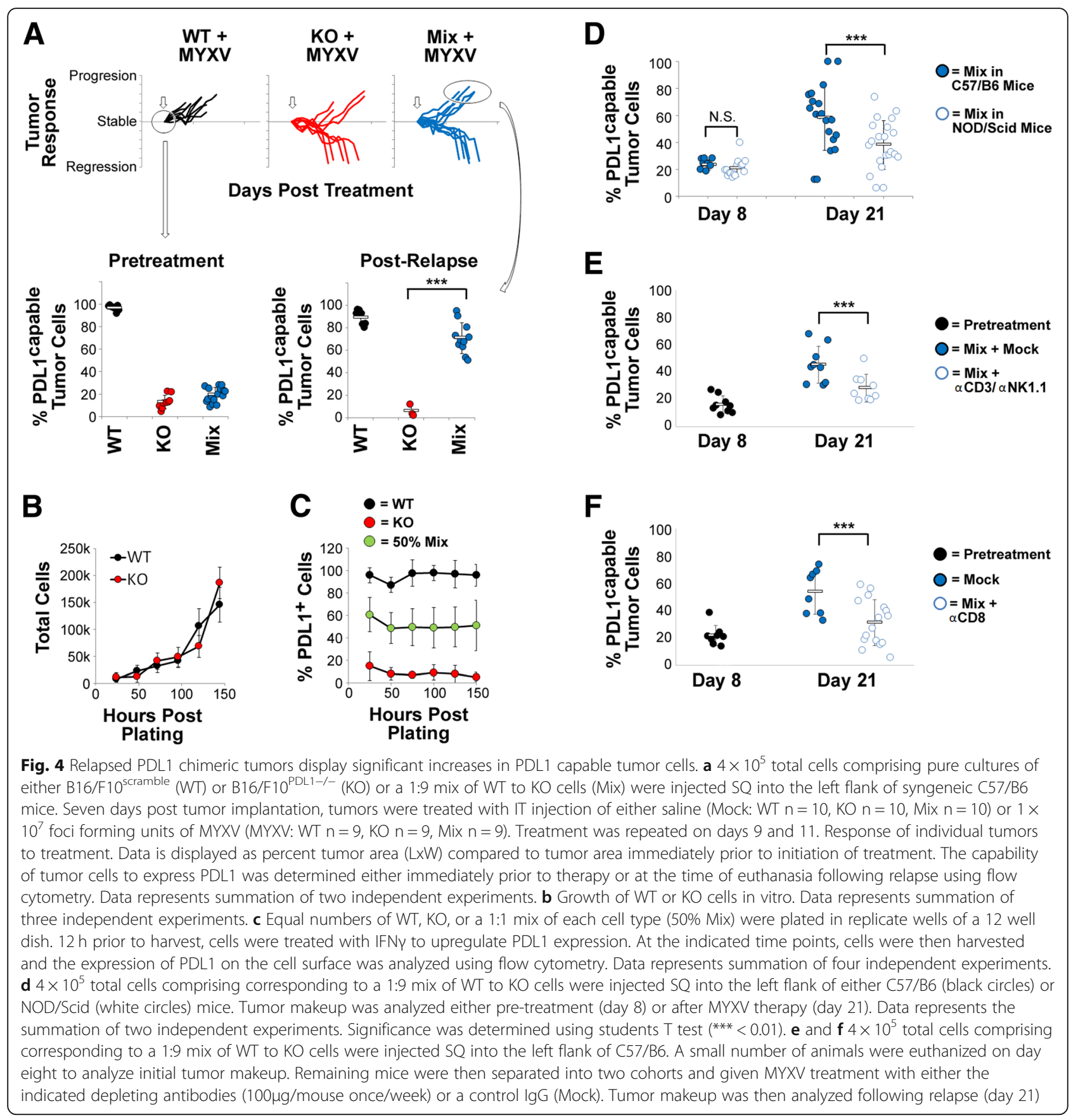

that the percentage of tumors cell capable of expressing PDL1 at the initiation of treatment displayed a strong inverse correlation with therapeutic outcome. Taken at face value, these data suggest that tumors expressing PDL1 in a limited number of tumor cells will likely relapse following $\mathrm{OV}$, however, these tumors will still gain more benefit from therapy than tumors expressing PDL1 on a high percentage of malignant cells. This conclusion, however, is subject to a number of caveats. First and foremost is that our use of CRISPR/Cas9 to genetically ablate expression of PDL1 prevents the inducible expression of this protein which is frequently seen following OV [28-30]. Our results must therefore be interpreted more in the context of constitutive PDL1 expression and not the context of inducible expression. Additionally, our studies also suffer from the typical limitations associated with rapidly progressing injectable tumor models. In our case, the foremost of these limitations is the inability to study the long-term impact of partial PDL1 positivity on anti-tumor immunity. Clinical tumors displaying constitutive PDL1 expression will have months or years for this partial positivity to impact anti-tumor immune function. In contrast, treatment 
in our model must be initiated within 7-10 days of tumor establishment due to the extremely rapid tumor progression. Low level PDL1 expression within a partially positive tumor might therefore impact anti-tumor immunity in ways not seen in our current studies. Finally, our studies were unable to identify the morphological architecture of our partially PDL1 positive tumors and it therefore remains unclear how the PDL1 ${ }^{+}$and $\mathrm{PDL}^{-}{ }^{-}$cells are distributed within each tumor. Critically, the nature of this distribution pattern might significantly influence the outcomes of treatment. Additional experiments are therefore required in order to more fully address this issue.

Interestingly, we observed that partially PDL1 positive tumors which relapsed following treatment displayed a significant increase in the percentage of PDL1 capable tumor cells (Fig. 4). This increase does not appear to be due to simple cellular growth rates since both the B16/F10 ${ }^{\text {scramble }}$ and B16/ F10 ${ }^{\text {PDL1- }-}$ cells used in our studies displayed identical growth rates (Fig. 4b) and selection for $\mathrm{PDL}^{+}$cells did not occur following mixed culture in vitro (Fig. 4c). Instead, our data suggests that this change in phenotype is due to active immunoediting and implicates $\mathrm{CD}^{+} \mathrm{T}$ cells in this process (Fig. 4). Interestingly, however, we routinely observed that loss of adaptive immunity reduced, but did not completely prevent, editing of our tumors (Fig. 4d-f). Therefore, while our data does implicate adaptive immune cells in this process, we cannot rule out other mechanisms such as intrinsic PDL1 survival signaling [31, 32]. Interestingly, previous work on intrinsic PDL1 signaling suggested that PDL1 deficient B16/F10 cells display an inherent growth defect in vitro compared to PDL1 capable cells, however, this defect was not observed in our studies (Fig. 4). The reason for this discrepancy is not clear; however, it has been shown that the intrinsic growth defects caused by ablation of PDL1 are amplified by both PD1 engagement and the presence of signaling molecules, such as interferon [31]. Neither of these factors was obviously present during our in vitro growth analysis which might explain the difference between our results and previous findings. Regardless, additional studies into the mechanism(s) mediating this editing and its impact on therapeutic outcomes therefore seem warranted.

\section{Methods}

\section{Cell lines and reagents}

B16/F10 (Cat\# CRL-6475) and BSC40 cell lines (Cat\# CRL-2761) were purchased from ATCC (Manassas, VA, USA). LLC and LLC-A9F1 cells were a kind gift form Dr. Mark Rubinstein. PDL1 deficient B16/F10 cells (B16/F10 PDL1 ${ }^{-/}$) and control B16/F10 cells treated with a scrambled gRNA (GCGAGGTATTCGGCTCCGCG) (B16/F10 ${ }^{\text {scramble }}$ ) were generated using the CRISPR/CAS9 system (Genscript, Piscataway, NJ, USA) as described previously [23]. MYXV (strain Lausanne) expressing GFP from an intergenic region between the $m 135 r$ and $m 136 r$ viral open reading frames
(vGFP) has been described elsewhere [23]. Cell viability was determined using the CellTiter-96 Non-Radioactive Cell Proliferation Assay (Promega, Madison, WI, USA) per manufacturer's recommendations. IFN $\gamma$ was purchased from Sino Biological (Wayne, PA, USA) and used at a concentration which was functionally titered for each lot. The following antibodies were used in these studies. For flow cytometry: PDL1 (clone MIH5), PD1 (clone J43), CD3 (clone 145-2c11), CD4 (clone RM4-5), CD8 (clone 53-6.7), CD69 (clone H1.2F3), CD25 (clone 3C7), CD45 (clone 30-F11), CD44 (clone IM7), F4/80 (clone T45-2342), and Ly6c (clone AL21). For western blot: PDL1 (Cat\# AF1019) (R\&D Systems, Minneapolis, MN, USA), and actin (clone I19) (Santa Cruz Biotechnology, Dallas, TX, USA). For immune depletion: CD3 (clone 145-2C11), CD8 (clone 53-6.7), NK1.1 (Clone PK136) (BioXcell, West Lebanon, NH).

\section{In vivo tumor models}

Six to eight week old C57/B6 (Charles River Laboratories,

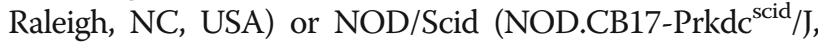
The Jackson Laboratory, Bar Harbor, ME, USA) mice were injected subcutaneously (SQ) with $4 \times 10^{5}$ total cells in $100 \mathrm{ul}$ low growth factor matrigel at a final concentration of $1.5 \mathrm{mg} / \mathrm{ml}$ (Corning, Corning, NY, USA). For mixed tumor studies, B16/F10 scramble and $\mathrm{B} 16 / \mathrm{F} 10^{\mathrm{PDL} 1-/-}$ cells were mixed at the indicated ratios ex vivo to give a total of $4 \times$ $10^{5}$ cells and the mixtures injected as above. Viral treatment consisted of three intratumoral injections of either saline or vGFP $\left(1 \times 10^{7}\right.$ total foci forming units (FFU) in 100ul PBS) on days 7, 9, and 11 post tumor implantation. For survival studies, tumor area was monitored using calipers and mice were euthanized when tumors reached 15 $\mathrm{mm}$ in any direction. For immunological and tumor analysis, tumors were excised, transferred onto a $40 \mu \mathrm{M}$ nylon mesh filter and mechanically separated into a single cell suspension and then stained for flow cytometry using standard methodologies [23]. To upregulate PDL1 expression on cells capable of expressing this protein, tumor cells were incubated with IFN $\gamma$ for $12 \mathrm{~h}$ prior to analysis. All analyses shown are pregated on single, viable events. All experiments were conducted in accordance with the Medical University of South Carolina Institutional Animal Care and Use Committee.

\section{Additional file}

Additional file 1: Figure S1. B16/F10 tumors lacking PDL1 are highly susceptible to oncolytic immunotherapy. Figure S2. Lung cancer tumors naturally lacking PDL1 are highly susceptible to oncolytic therapy. Figure S3. Lack of PDL1 does not influence MYXV infection. (PDF 358 kb)

\section{Acknowledgments}

We thank Dr. Mark Rubinstein and Dr. Chrystal Paulos for valuable discussion of this project. 


\section{Funding}

This work was supported by grants to Dr. Eric Bartee from: NIH-NIAID (1K22Al095372-01A1), NIH-NCI (1R01CA194090-01A1), ACS (RSG-17-047-01MPC), and intramural funding from the Medical University of South Carolina. This work was also supported in part by the Hollings Cancer Center's Support Grant P30 CA138313.

\section{Availability of data and materials}

No large data sets are presented in the current manuscript. All materials needed to reproduce the current experiments are commercially available or will be shared with other investigators.

\section{Authors' contributions}

EB: Designed, conducted, and analyzed experiments regarding impact of PDL1 heterogeneity in vivo, prepared manuscript, MB: generated PDL1 KO and control cell lines and conducted experiments regarding infection of MYXV in these cell lines, PCD: conducted experiments and collected data concerning growth rate of cell in vitro. All authors read and approved the final manuscript.

\section{Ethics approval and consent to participate}

Not Applicable.

\section{Consent for publication}

Not Applicable.

\section{Competing interests}

The authors declare that they have no competing interests.

\section{Publisher's Note}

Springer Nature remains neutral with regard to jurisdictional claims in published maps and institutional affiliations.

\section{Received: 22 August 2018 Accepted: 26 December 2018}

\section{Published online: 16 January 2019}

\section{References}

1. Riley JL. PD-1 signaling in primary T cells. Immunol Rev. 2009;229(1):114-25.

2. Blank C, Mackensen A. Contribution of the PD-L1/PD-1 pathway to T-cel exhaustion: an update on implications for chronic infections and tumor evasion. Cancer Immunol Immunother. 2007;56(5):739-45.

3. Dong H, Strome SE, Salomao DR, Tamura H, Hirano F, Flies DB, et al. Tumorassociated B7-H1 promotes T-cell apoptosis: a potential mechanism of immune evasion. Nat Med. 2002:8(8):793-800.

4. Dong H, Chen L. B7-H1 pathway and its role in the evasion of tumor immunity. J Mol Med. 2003:81(5):281-7.

5. Oguejiofor K, Galletta-Williams H, Dovedi SJ, Roberts DL, Stern PL, West CM. Distinct patterns of infiltrating CD8+ T cells in HPV+ and CD68 macrophages in HPV- oropharyngeal squamous cell carcinomas are associated with better clinical outcome but PD-L1 expression is not prognostic. Oncotarget. 2017;8(9):14416-27.

6. Lu C, Redd PS, Lee JR, Savage N, Liu K. The expression profiles and regulation of PD-L1 in tumor-induced myeloid-derived suppressor cells. Oncoimmunology. 2016;5(12):e1247135.

7. Huang ZY, Xu P, Li JH, Zeng CH, Song HF, Chen H, et al. Clinical significance of dynamics of programmed death Ligand-1 expression on circulating CD14(+) monocytes and CD19(+) B cells with the progression of hepatitis B virus infection. Viral Immunol. 2017;30(3):224-31.

8. Zhang Y, Zhu W, Zhang X, Qu Q, Zhang L. Expression and clinical significance of programmed death-1 on lymphocytes and programmed death ligand-1 on monocytes in the peripheral blood of patients with cervical cancer. Oncol Lett. 2017;14(6):7225-31.

9. Dieterich LC, Ikenberg K, Cetintas T, Kapaklikaya K, Hutmacher C, Detmar M. Tumor-associated lymphatic vessels upregulate PDL1 to inhibit T-cell activation. Front Immunol. 2017;8:66.

10. He H, Qi L, Xiao Y, Hou Y. Tumor Associated Fibroblasts Promote PD-L1 Expression in Lung Cancer Cells. Zhongguo fei ai za zhi = Chinese journal of lung cancer. 2017:20(5):293-7.

11. Casadevall D, Clave S, Taus A, Hardy-Werbin M, Rocha P, Lorenzo M, et al. Heterogeneity of tumor and immune cell PD-L1 expression and lymphocyte counts in surgical NSCLC samples. Clin Lung Cancer. 2017;18(6):682-91 e5.
12. Tarhini AA, Zahoor H, Yearley JH, Gibson C, Rahman Z, Dubner R, et al. Tumor associated PD-L1 expression pattern in microscopically tumor positive sentinel lymph nodes in patients with melanoma. J Transl Med. 2015:13:319.

13. Lee LH, Cavalcanti MS, Segal NH, Hechtman JF, Weiser MR, Smith JJ, et al. Patterns and prognostic relevance of PD-1 and PD-L1 expression in colorectal carcinoma. Mod Pathol. 2016;29(11):1433-42.

14. Pinto N, Park JR, Murphy E, Yearley J, McClanahan T, Annamalai L, et al. Patterns of PD-1, PD-L1, and PD-L2 expression in pediatric solid tumors. Pediatr Blood Cancer. 2017;64:11.

15. Thompson ED, Zahurak M, Murphy A, Cornish T, Cuka N, Abdelfatah E, et al. Patterns of PD-L1 expression and CD8 T cell infiltration in gastric adenocarcinomas and associated immune stroma. Gut. 2017:66(5):794-801.

16. Scognamiglio T, Chen YT. Beyond the percentages of PD-L1-positive tumor cells: induced versus constitutive PD-L1 expression in primary and metastatic head and neck squamous cell carcinoma. Head Neck Pathol. 2017;12(2):221-9.

17. Taube JM, Anders RA, Young GD, Xu H, Sharma R, McMiller TL, et al. Colocalization of inflammatory response with B7-h1 expression in human melanocytic lesions supports an adaptive resistance mechanism of immune escape. Sci Transl Med. 2012;4(127):127ra37.

18. Liang M, Yang H, Fu J. Nimesulide inhibits IFN-gamma-induced programmed death-1-ligand 1 surface expression in breast cancer cells by COX-2 and PGE2 independent mechanisms. Cancer Lett. 2009;276(1):47-52.

19. Azuma K, Ota K, Kawahara A, Hattori S, Iwama E, Harada T, et al. Association of PD-L1 overexpression with activating EGFR mutations in surgically resected nonsmall-cell lung cancer. Ann Oncol. 2014;25(10):1935-40.

20. Parsa AT, Waldron JS, Panner A, Crane CA, Parney IF, Barry JJ, et al. Loss of tumor suppressor PTEN function increases B7-H1 expression and immunoresistance in glioma. Nat Med. 2007;13(1):84-8.

21. Melcher A, Parato K, Rooney CM, Bell JC. Thunder and lightning: immunotherapy and oncolytic viruses collide. Mol Ther. 2011;19(6):1008-16.

22. de Gruij TD, Janssen AB, van Beusechem WW. Arming oncolytic viruses to leverage antitumor immunity. Expert Opin Biol Ther. 2015;15(7):959-71.

23. Bartee MY, Dunlap KM, Bartee E. Tumor-localized secretion of soluble PD1 enhances oncolytic Virotherapy. Cancer Res. 2017;77(11):2952-63.

24. Madore J, Vilain RE, Menzies AM, Kakavand H, Wilmott JS, Hyman J, et al. PD-L1 expression in melanoma shows marked heterogeneity within and between patients: implications for anti-PD-1/PD-L1 clinical trials. Pigment Cell Melanoma Res. 2015;28(3):245-53.

25. Robert C. Checkpoint blockade plus oncolytic virus: a hot therapeutic Cancer strategy. Trends Mol Med. 2017;23(11):983-5.

26. Kim HR, Ha SJ, Hong MH, Heo SJ, Koh YW, Choi EC, et al. PD-L1 expression on immune cells, but not on tumor cells, is a favorable prognostic factor for head and neck cancer patients. Sci Rep. 2016;6:36956.

27. Juneja VR, McGuire KA, Manguso RT, LaFleur MW, Collins N, Haining WN, et al. PD-L1 on tumor cells is sufficient for immune evasion in immunogenic tumors and inhibits CD8 T cell cytotoxicity. J Exp Med. 2017;214(4):895-904.

28. Liu Z, Ravindranathan R, Kalinski P, Guo ZS, Bartlett DL. Rational combination of oncolytic vaccinia virus and PD-L1 blockade works synergistically to enhance therapeutic efficacy. Nat Commun. 2017;8:14754.

29. Samson A, Scott KJ, Taggart D, West EJ, Wilson E, Nuovo GJ, et al. Intravenous delivery of oncolytic reovirus to brain tumor patients immunologically primes for subsequent checkpoint blockade. Sci Transl Med. 2018:10:422.

30. Zamarin D, Ricca JM, Sadekova S, Oseledchyk A, Yu Y, Blumenschein WM, et al. PD-L1 in tumor microenvironment mediates resistance to oncolytic immunotherapy. J Clin Invest. 2018;128(11):5184.

31. Gato-Canas M, Zuazo M, Arasanz H, Ibanez-Vea M, Lorenzo L, FernandezHinojal G, et al. PDL1 signals through conserved sequence motifs to overcome interferon-mediated cytotoxicity. Cell Rep. 2017;20(8):1818-29.

32. Clark CA, Gupta HB, Sareddy G, Pandeswara S, Lao S, Yuan B, et al. Tumorintrinsic PD-L1 signals regulate cell growth, pathogenesis, and autophagy in ovarian Cancer and melanoma. Cancer Res. 2016;76(23):6964-74. 